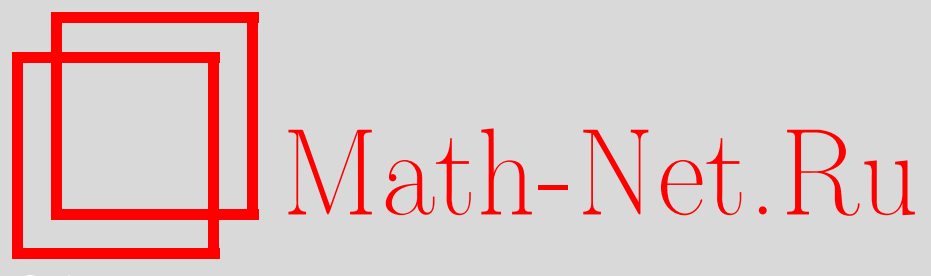

А. В. Клименко, Конечность числа классов марковских разбиений для псевдоаносовских диффеоморфизмов поверхностей, Матем. заметки, 2009, том 86, выпуск $2,314-317$

DOI: https://doi.org/10.4213/mzm8478

Использование Общероссийского математического портала Math-Net.Ru подразумевает, что вы прочитали и согласны с пользовательским соглашением http://www . mathnet.ru/rus/agreement

Параметры загрузки:

IP: 54.224 .60 .19

26 апреля 2023 г., 12:03:49

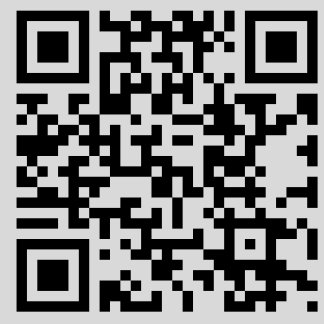




\section{Конечность числа классов марковских разбиений для псевдоаносовских диффеоморфизмов поверхностей}

\section{А. В. Клименко}

Одним из средств для изучения динамики псевдоаносовских диффеоморфизмов (определение см. $[1 ;$ гл. 6]) является построение сопряжения такого диффеоморфизма и марковской цепи. (С метрической точки зрения это "настоящее" сопряжение, с топологической - нет: точкам из некоторого нигде не плотного множества соответствует более одной последовательности в марковской цепи.) Такое построение для случая аносовского диффеоморфизма двумерного тора сделано впервые Адлером и Вейссом в работе [2] и изложено, например, в книге [3]. В общем случае псевдоаносовского диффеоморфизма это описано в книге [4]. Далее мы предполагаем фиксированным псевдоаносовский диффеоморфизм $T$ поверхности $M$.

Существуют различные определения марковского разбиения $\Xi=\left(X_{1}, \ldots, X_{m}\right)$. Вопервых, можно было бы просто требовать, чтобы индуцированная разбиением мера на $\{1, \ldots, m\}^{\mathbb{Z}}$ являлась марковской. Такое определение слишком широко. В частности, согласно Орнстейну [5] индуцированная мера может быть сделана бернуллиевской. Кроме того, из этого определения не видно, как можно было бы строить марковские разбиения.

Более узкое определение принадлежит Боуэну (см. [6], [7; §3С], [3; §18.7]). Говоря неформально, каждое из множеств $X_{i}$ является "прямоугольником" - прямым произведением в смысле теоремы о локальном прямом произведении, при этом каждый устойчивый слой прямоугольника под действием отображения вкладывается в некоторый устойчивый слой (возможно, другого) прямоугольника; для неустойчивых слоев верно то же для обратного отображения. Можно показать, что уже для аносовского диффеоморфизма тора существует бесконечно много различных (несводимых друг к другу применением отображения) марковских разбиений в смысле Боуэна.

Изначальное определение Адлера и Вейсса для диффеоморфизма тора отличается тем, что $X_{i}$ являются не просто прямыми произведениями, а "геометрическими" прямоугольниками (точнее, параллелограммами). При этом, как и в более общем боуэновском случае, если параллелограммы достаточно мелки, кодирование является взаимно однозначным почти всюду. Такое измельчение всегда можно получить (см. [4; 10]), беря в качестве элементов разбиения связные компоненты множеств $\operatorname{clos}\left(\operatorname{int} X_{i_{0}} \cap \operatorname{int} T^{-1}\left(X_{i_{1}}\right) \cap\right.$ $\left.\cdots \cap \operatorname{int} T^{-k}\left(X_{i_{k}}\right)\right)$, где $k$ фиксировано и достаточно велико, а $X_{j}$ образуют предмарковское разбиение (см. ниже). Мы используем этот термин, следуя [4], в других источниках (например, в [1]) такие разбиения называются марковскими; в отличие от этого, в [4] так называют лишь предмарковские разбиения, порождающие почти однозначное кодирование.

ОПРеДЕЛЕниЕ 1. Предмарковским разбиением называется разбиение

$$
\Xi=\left(X_{1}, \ldots, X_{m}\right)
$$

которое удовлетворяет следующим свойствам:

1) каждое $X_{j}$ является параллелограммом, т.е. существует непрерывное отображение $h_{j}:[0,1]^{2} \rightarrow X_{j}$ такое, что его ограничение на $(0,1)^{2}$ является гомеоморфизмом, $h_{j}([0,1] \times\{y\})$ при всех $y \in(0,1)$ является отрезком слоя устойчивого слоения $\mathscr{F}^{\mathrm{s}}$ для $T$, а $h_{j}(\{y\} \times[0,1])$ при $x \in(0,1)$ - отрезком слоя неустойчивого слоения $\mathscr{F}^{\mathrm{u}}$;

Работа выполнена при поддержке Российского фонда фундаментальных исследований (грант № 08-01-00342-а) и программы "Ведущие научные школы" (грант № НШ-3038.2008.1). 
множество $h_{j}(\{0,1\} \times[0,1])$ будем называть неустойчивой гранищей $X_{j}$ и обозначать через $\partial^{\mathrm{u}} X_{j}$; аналогично, $h_{j}([0,1] \times\{0,1\})$ будем называть его устойчивой гранищей $\partial^{\mathrm{s}} X_{j}$; также положим $\partial^{\mathrm{u}, \mathrm{s}} \Xi=\bigcup_{j} \partial^{\mathrm{u}, \mathrm{s}} X_{j}, \partial \Xi=\partial^{\mathrm{s}} \Xi \cup \partial^{\mathrm{u}} \Xi$;

2) $T\left(\partial^{\mathrm{u}} \Xi\right) \supset \partial^{\mathrm{u}} \Xi, T\left(\partial^{\mathrm{s}} \Xi\right) \subset \partial^{\mathrm{s}} \Xi$.

Встает вопрос о количестве различных предмарковских разбиений. Очевидно, что имея одно предмарковское разбиение $\Xi$, можно получить бесконечную серию таких разбиений $T^{n}(\Xi), n \in \mathbb{Z}$. Поэтому естественно дать следующее определение.

ОПредЕЛЕниЕ 2. Разбиения $\Xi^{(1)}$ и $\Xi^{(2)}$ называются эквивалентными, если $\Xi^{(2)}=$ $T^{k}\left(\Xi^{(1)}\right)$ при некотором целом $k$.

Теорема. Число классов эквивалентности предмарковских разбиений с фиксированным количеством элементов разбиения конечно.

Отметим, что можно рассматривать и более широкую эквивалентность: $\Xi^{(1)} \sim \Xi^{(2)}$, если $\Xi^{(2)}=S\left(\Xi^{(1)}\right)$ для некоторого гомеоморфизма $S$, сохраняющего устойчивое и неустойчивое слоения и коммутирующего с $T$. Ясно, что число классов эквивалентности для такого определения будет не больше, чем для приведенного выше.

ДоКАЗАТЕЛЬСТво тЕОРемы. 1. Пусть $\Xi$ - произвольное разбиение из $m$ элементов, $A^{\mathrm{s}, \mathrm{u}}$ - множества слоев устойчивого и неустойчивого слоений, дуги которых входят в $\partial \Xi$. Докажем, что все слои $A^{\mathrm{s}}$ являются периодическими с периодом не более $4 \mathrm{~m}$. Для этого, так как $T\left(A^{\mathrm{s}}\right)=A^{\mathrm{s}}$, достаточно доказать, что $A^{\mathrm{s}}$ содержит не более $4 \mathrm{~m}$ слоев. Если это не так, некоторое множество $h_{j}([0,1] \times\{i\}), j=1, \ldots, m, i=0,1$, содержит отрезки по меньшей мере трех слоев, т.е. кривая $\gamma_{i, j}(t)=h_{j}(t, i)$ как минимум дважды проходит через множество $\operatorname{Sing}(T)$ особых точек слоений. Выбрав $s_{1,2}$ так, что

$$
\gamma_{i, j}\left(s_{1,2}\right) \in \operatorname{Sing}(T), \quad \gamma_{i, j}\left(\left(s_{1}, s_{2}\right)\right) \cap \operatorname{Sing}(T)=\varnothing,
$$

получим, что $\gamma_{i, j}\left(\left[s_{1}, s_{2}\right]\right)$ является слоем, соединяющим две особые точки, а таких слоев для псевдоаносовского диффеоморфизма не может быть. Итак, $A^{\mathrm{s}}$ является подмножеством множества $P_{4 m}^{\mathrm{s}}$ всех устойчивых слоев периода не более $4 m$. Аналогично, $A^{\mathrm{u}} \subset P_{4 m}^{\mathrm{u}}$.

2. Заметим, что $T^{q}$ переводит слой $\beta \in A^{\mathrm{s}}$ периода $q$ в себя, сжимая его. Более того, $T^{q}$ переводит отрезок $\beta \cap \partial^{\mathrm{s}} \Xi$ внутрь себя, поэтому на нем есть периодическая точка периода $q$.

Введем на слое $\beta \in P_{4 m}^{\mathrm{s}, \mathrm{u}}$ параметризацию, которую обозначим $\beta(t)$, так, что $\beta(0)-$ это периодическая точка, а $\mu_{\mathrm{s}, \mathrm{u}}(\beta([a, b]))=|b-a|$. Такая параметризация единственна с точностью до замены знака, причем $t \in(-\infty, \infty)$, если $\beta(0)$ неособая, и $t \in[0,+\infty)$ или $t \in(-\infty, 0]$, если она особая; в последнем случае для удобства доопределим $\beta(t)$ по правилу $\beta(t)=\beta(-t)$.

Число пересечений (не примыканий) в неособых точках отрезков границы разбиения, не превосходит $m$. Действительно, каждой такой точке соответствует 4 угла параллелограммов разбиения, а общее число углов равно $4 m$. В силу леммы 1 (см. ниже) существует такая константа $L$, что либо

$$
\partial^{\mathrm{s}} \Xi \subset D_{4 m}^{\mathrm{s}}(1):=\bigcup_{\beta \in P_{4 m}^{\mathrm{s}}} \beta([-1,1])
$$

либо

$$
\partial^{\mathrm{u}} \Xi \subset D_{4 m}^{\mathrm{u}}(L):=\bigcup_{\gamma \in P_{4 m}^{\mathrm{u}}} \gamma([-L, L]) .
$$

(Надо взять $L$ таким, что каждый из отрезков $\gamma([0, \pm L])$ пересекает каждый $\beta([0, \pm 1])$ не менее $m+1$ раза.) 
3. Для данного $\Xi$ выберем $k$ так, что $\partial^{\mathrm{s}}\left(T^{k} \Xi\right) \subset D_{4 m}^{\mathrm{s}}(1 / \lambda)$, но $\partial^{\mathrm{s}}\left(T^{k} \Xi\right) \not \subset D_{4 m}^{\mathrm{s}}(1)$, где $\lambda<1$ - коэффициент сжатия на слоях. Тогда $\partial^{\mathrm{u}}\left(T^{k} \Xi\right) \subset D_{4 m}^{\mathrm{u}}(L)$. Итак, в каждом классе эквивалентности $[\Xi]$ можно выбрать такого представителя $\Xi^{*}$, что

$$
\partial^{\mathrm{u}} \Xi^{*} \subset D_{4 m}^{\mathrm{u}}(L), \quad \partial^{\mathrm{s}} \Xi^{*} \subset D_{4 m}^{\mathrm{s}}\left(\frac{1}{\lambda}\right)
$$

Число разбиений, удовлетворяющих этим условиям, конечно. Действительно, для каждого $\beta \in P_{4 m}^{\mathrm{s}}$ множество $D_{4 m}^{\mathrm{u}}(L) \cap \beta([-1 / \lambda, 1 / \lambda])$ конечно. Но концы отрезка слоя, входящего в границу разбиения, обязаны принадлежать этому множеству. Следовательно, число способов выбрать каждый отрезок границы разбиения конечно, а тогда и общее число таких разбиений конечно. Теорема доказана.

Лемма 1. Любой полуслой периодического слоя $\mathscr{F}^{\mathrm{u}, \mathrm{s}}$ с началом в периодической точке всюду плотен. ${ }^{1}$

ДокАзАтЕльство. 1. Для определенности будем рассматривать полуслой $\beta^{+}=\beta((0$, $+\infty)$ ) на неустойчивом слое $\beta$. Если $\beta$ имеет период $q$ под действием $T$, то $\widehat{T}=T^{2 q}$ оставляет полуслой $\beta^{+}$неподвижным.

Положим $M_{0}=M \backslash(\{\beta(0)\} \cup \operatorname{Sing}(T))$. Множество $M_{0}$ является связным $\widehat{T}$-инвариантным множеством. Мы покажем, что $\cos _{M_{0}} \beta^{+}=M_{0}$, тогда, очевидно, $\cos _{M} \beta^{+}=M$. Учитывая связность $M_{0}$, для этого достаточно доказать, что $\cos _{M_{0}} \beta^{+}$открыто в нем.

2. Пусть $x \in \cos _{M_{0}} \beta^{+}$. Выберем окрестность $U \subset M_{0}$ и выпрямляющие координаты $(\xi, \eta)$ в ней так, что $U=\left\{|\xi|<\xi_{0},|\eta|<\eta_{0}\right\}, x$ имеет координаты $(0,0)$, отрезки слоев $\mathscr{F}^{\text {u }}$ (соответственно $\left.\mathscr{F}^{\mathrm{s}}\right)$ в $U$ являются горизонтальными отрезками $\left\{\eta=\eta^{*}\right\}$ (соответственно вертикальными $\left\{\xi=\xi^{*}\right\}$ ), причем трансверсальные меры на этих слоях таковы, что

$$
\mu_{\mathrm{u}}\left(\left[\xi_{1}, \xi_{2}\right] \times\left\{\eta^{*}\right\}\right)=\left|\xi_{2}-\xi_{1}\right|, \quad \mu_{\mathrm{s}}\left(\left\{\xi^{*}\right\} \times\left[\eta_{1}, \eta_{2}\right]\right)=\left|\eta_{2}-\eta_{1}\right| .
$$

Поскольку $\beta(0)$ не содержится в $U, \cos _{M_{0}} \beta^{+}$пересекает $U$ по семейству интервалов $\left(-\xi_{0}, \xi_{0}\right) \times A$, где $A-$ подмножество интервала $\left(-\eta_{0}, \eta_{0}\right)$, замкнутое в нем.

3. Докажем, что $U \subset \operatorname{clos}_{M_{0}} \beta^{+}$. Пусть $y=\left(\xi_{y}, \eta_{y}\right) \in U$. Положим $\varepsilon=\operatorname{dist}\left(\eta_{y}, A\right)$. Если $\varepsilon=0$, то в силу замкнутости $A \quad y \in \cos _{M_{0}} \beta^{+}$, в противном случае существует $\eta^{\prime} \in A,\left|\eta_{y}-\eta^{\prime}\right|=\varepsilon$. Положим

$$
\mu=\min \left(\varepsilon \frac{1-\lambda}{1+\lambda}, \eta_{0}-\eta_{y}, \eta_{y}+\eta_{0}\right) .
$$

Поскольку у неблуждающая, существует $z=\left(\xi_{z}, \eta_{z}\right) \in U$ и $r \geqslant 1$, что $z, w=\widehat{T}^{r}(z) \in$ $\left(\xi_{y}-\mu, \xi_{y}+\mu\right) \times\left(\eta_{y}-\mu, \eta_{y}+\mu\right)$. Точки $z$ и $z^{\prime}=\left(\xi_{z}, \eta^{\prime}\right) \in \cos _{M_{0}} \beta^{+}$лежат на одном и том же устойчивом слое и мера отрезка между ними не превосходит $\left|\eta_{z}-\eta_{y}\right|+\left|\eta_{y}-\eta^{\prime}\right| \leqslant \varepsilon+\mu$. Тогда точка $\widehat{T}^{r} z=w=\left(\xi_{w}, \eta_{w}\right)$ и $\widehat{T}^{r} z^{\prime}=w^{\prime}$ также лежат на одном устойчивом слое и мера отрезка между ними не больше $\lambda(\varepsilon+\mu)$. Поскольку $w^{\prime} \in \cos _{M_{0}} \beta^{+}, \operatorname{dist}\left(\eta_{w}, A\right) \leqslant$ $\lambda(\varepsilon+\mu)$. Тогда

$$
\operatorname{dist}\left(\eta_{y}, A\right) \leqslant\left|\eta_{y}, \eta_{w}\right|+\operatorname{dist}\left(\eta_{w}, A\right)<\lambda \varepsilon+(\lambda+1) \mu \leqslant \varepsilon,
$$

что противоречит тому, что $\operatorname{dist}\left(\eta_{y}, A\right)=\varepsilon$.

\footnotetext{
${ }^{1}$ Данное утверждение, несомненно, известно (и даже в более общем виде - для любого полуслоя на любом слое, за исключением "полуслоя", ограниченного с одной стороны некоторой точкой $a$, а с другой стороны особой точкой). Однако мы не смогли дать соответствующую ссылку и потому приводим доказательство.
} 


\section{СПИСОК ЦИТИРОВАННОЙ ЛИТЕРАТУРЫ}

[1] Э. Кэссон, С. Блейлер, Теория автоморфизмов поверхностей по Нильсену и Тёрстону, Библиотека студента-математика, 5, ФАЗИС, М., 1998. [2] R.L. Adler, B. Weiss, Proc. Natl. Acad. Sci. USA, 57:6 (1967), 1573-1576. [3] А. Б. Каток, Б. Хасселблат, Введение в современную теорию динамических систем, Факториал, М., 1999. [4] A. Fathi, F. Laudenbach, V. Poenaru, Travaux de Thurston sur les surfaces. Séminaire Orsay, Astérisque, 66-67, Soc. Math. France, Paris, 1979. [5] D. Ornstein, Adv. in Math., 4:3 (1970), 337-352. [6] R. Bowen, Amer. J. Math., 92:3 (1970), 725-747. [7] Р. Боуэн, Методы символической динамики, Сб. статей, Математика. Новое в зарубежной науке, 13, Мир, М., 1979, 9-92.

А. В. Клименко

Поступило Математический институт им. В. А. Стеклова РАН

E-mail: klimenko05@mail.ru 